\title{
Two-Quantum Photoprocesses in DNA and RNA Biopolymers Under Powerful Picosecond Laser UV Irradiation
}

\author{
DAVID N. NIKOGOSYAN $\dagger$
}

Institute of Spectroscopy, USSR Academy of Sciences, Troitzk, Moscow Region, 142092, USSR

and

GAGIK G. GURZADYAN

Institute for Physical Research, Armenian SSR Academy of Sciences, Ashtarak, Armenian SSR, 378410, USSR

Two-quantum photoreactions taking place under picosecond laser UV irradiation of DNA and RNA biopolymers were investigated. Despite of qualitative difference in two-step photoprocesses concerned (single-strand breaks formation in plasmid pBR 322 DNA, induction of breaks in TMV RNA sugar-phosphate chain, cross-links formation in DNA of $C_{D}$ phage) similar dependences of the quantum yield of the effects studied on the irradiation intensity can be observed with saturation in the region of $3 \cdot 10^{11}-3 \cdot 10^{12} \mathrm{~W} / \mathrm{m}^{2}$. The saturation intensity is one or two orders lower than in the case of irradiation of single monomers - nucleic acid bases.

\section{INTRODUCTION}

It is well known that nucleic acid biopolymers-DNA and RNA consist of chromophores, or nucleic acid bases absorbing in the UV spectral range with maximum near $250-270 \mathrm{~nm}$, and sugar-phosphate chains absorbing in a shorter-wave UV range (shorter than $220 \mathrm{~nm}$ ). Lowintensity UV irradiation $\left(I=10^{-1}-10^{1} \mathrm{~W} / \mathrm{m}^{2}, \lambda=254 \mathrm{~nm}\right)$ of DNA and RNA, nucleic acid components including bases, as well as the

† To whom all correspondence should be addressed. 
bases themselves leads to one-quantum (one-step) excitation of lowlying electronic levels $S_{1}$ and $T_{1}$ of the base molecules due to absorption of one radiation quantum. As a result, one-quantum photochemical reactions are realized, among which dimerization and hydratation of pyrimidine bases are dominant. ${ }^{1}$

In powerful laser UV irradiation of the isolated bases in aqueous solution $\left(I>10^{11} \mathrm{~W} / \mathrm{m}^{2}, \lambda=266 \mathrm{~nm}\right)$ the population of the states $S_{1}$ and $T_{1}$ as well as the rate of these levels' depletion are sharply increased due to transitions into high-lying electronic levels $S_{N}$ and $T_{N}$. Thus, two-step excitation of the electronic levels $S_{N}$ (or $T_{N}$ ) is realized through an intermediate step $-S_{1}\left(T_{1}\right)$ state. ${ }^{2,3}$ It was shown, that at picosecond UV irradiation the two-step excitation of bases takes place through the singlet channel (second quantum is absorbed in $S_{1}$ state); at nanosecond UV irradiation-through the triplet channel (second quantum is absorbed in $T_{1}$ state) ${ }^{4}$ In both cases the excited electronic $S_{N}$ and $T_{N}$ levels are above the ionization threshold of the bases in aqueous solution $(\sim 5.5-6.5 \mathrm{eV}),{ }^{5}$ as a result of which the base molecules are ionized and chemical reactions with high quantum yield are realized.

By quantum yield of photoreaction (both single-quantum and twoquantum) we mean a ratio of a number of molecules being reacted to a number of absorbed radiation quanta. Due to such a determination of the quantum yield it is possible to compare the quantum efficiencies of one-quantum and two-quantum photoreactions as well as to elucidate their relative contribution using their dependences on radiation intensity.

It is well known that in a classical case of low-intensity UV irradiation the quantum yield of single-quantum photoreaction is constant and it does not depend on radiation intensity (Bunsen-Roscolaw). ${ }^{6}$ At the same time quantum yield of two-quantum photoreaction increases with the intensity growth up to saturation of one of the steps of two-step excitation process. ${ }^{4}$

It should be noted that in high-intensity UV irradiation the quantum vield of single-quantum photoreactions from $S_{1}$ and $T_{1}$ levels also art to depend on intensity, namely, decrease inversely proportional radiation intensity. ${ }^{7}$ This takes place when the rate constants of $S_{1}$

' $T_{1}$ depletion levels due to $S_{1} \rightarrow S_{N}$ and $T_{1} \rightarrow T_{N}$ transitions begin exceed the sum of all other rate constants of $S_{1}$ and $T_{1}$ levels seactivation. 
Thus, at powerful laser UV irradiation of the isolated nucleic acid bases the two-step excitation of high-lying $S_{N}$ and $T_{N}$ electronic states is realized. With UV radiation intensity growth the quantum yields of two-quantum photoreactions increase up to saturation of one of the step of two-step excitation process, and quantum yields of singlequantum photoreactions fall.

We revealed a new effect which comes about as follows. At two-step excitation of bases in nucleic acid biopolymers the quantum yields of two-quantum photoreactions are saturated at UV irradiation intensities by one or two orders of magnitude lower than in case of irradiation of the separate isolated bases. This effect also results in simultaneous decreasing of the intensity value from which the quantum yield of one-quantum photoreaction of bases in the nucleic acid biopolymers starts to fall. The revealed effect is demonstrated below for some instances and a qualitative interpretation of the effect is given.

\section{MATERIALS AND METHODS}

In the experiments described a picosecond Nd:YAG laser with frequency conversion into the fourth harmonic $(\lambda=266 \mathrm{~nm})^{8}$ was used as the source of powerful laser UV radiation. The UV radiation parameters are: energy $10 \mathrm{~mJ}$, pulse duration $30 \mathrm{ps}$, laser beam crosssection $0.3 \mathrm{~cm}^{2}$, pulse repetition rate $0.5 \mathrm{~Hz}$. The radiation intensity was varied by means of lens within $10^{10}-10^{14} \mathrm{~W} / \mathrm{m}^{2}$. A low pressure mercury lamp $\left(\lambda=254 \mathrm{~nm}, I=1 \mathrm{~W} / \mathrm{m}^{2}\right)$ was used as the source of low-intensity UV radiation.

The photoreaction of single-strand breaks formation in plasmid pBR 322 DNA was investigated by electrophoresis in agarose gel. ${ }^{9,10}$ The concentration of DNA aqueous solution was $10 \mu \mathrm{g} / \mathrm{ml}$, optical density in $0.5 \mathrm{~cm}$ cell was $D=0.1$. The breaks formation of the sugar-phosphate chain of TMV RNA was investigated by a method of analytical centrifugation. ${ }^{11}$ The optical density of RNA aqueous solution was $D=6$ in the $0.5 \mathrm{~cm}$ cell (concentration $0.5 \mathrm{mg} / \mathrm{ml}$ ). Formation of the intramolecular cross-links in phage $C_{D}$ DNA was studied by luminescence technique using acridine orange dye. The concentration of DNA solution was $15 \mu \mathrm{g} / \mathrm{ml}, D=0.15$ in the $0.5 \mathrm{~cm}$ cell. Two-quantum photoreaction of thymine base decomposition in concentrated aqueous solution $\left(C=10^{-3} \mathrm{M}, L=0.1 \mathrm{~cm}\right)$ was investi- 
gated by means of absorption UV spectrophotometry. ${ }^{4}$ We have also studied one-quantum photoreaction of the thymine dimer formation in polynucleotide poly-dT by means of reversion of the pyrimidine dimers formed into monomers. ${ }^{7}$ The poly-dT samples with optical density $D \approx I$ in the $1 \mathrm{~cm}$ cell were used.

\section{RESULTS AND DISCUSSIONS}

Figure 1 represents the experimental dependences of quantum yields of the single-strand breaks formation in plasmid pBR 322 DNA, sugar-phosphate chain breaks induction in TMV RNA, cross-links formation in phase $C_{D}$ DNA and photolysis of concentrated aqueous thymine solution on the intensity of picosecond laser irradiation. It is seen that with the rise of radiation intensity the increase of quantum yields of all the reactions investigated followed by saturation takes place, which confirms two-step mechanism of these photolesions formation.

From Figure 1 it is seen that all two-quantum photoreactions dealing with the bases excitation in biopolymers have the qualitatively similar quantum yield dependences on intensity. This is explained by the fact, that the primary excitation channel in all cases is the same and is associated with excitation of chromophores-nucleic acid bases. It is important to indicate that the intensity value, corresponding to saturation of the quantum yield dependence, in case of biopolymer irradiation is by one-two orders lower than the saturation intensity in case of two-quantum photolysis of the isolated DNA base-thymine.

Similar phenomenon is also observed while investigating onequantum photoreactions in the nucleic acid biopolymers under powerful laser UV irradiation. Dependence of the quantum yield of thymine dimer formation in poly-dT on the irradiation intensity is represented in Figure 2. It is plotted on the base of experimental data obtained in. ${ }^{7}$ It is seen that fall of quantum yield of one-quantum photoreaction in poly-dT begins with $I \approx 2 \cdot 10^{12} \mathrm{~W} / \mathrm{m}^{2}$. At the same time the calculations made on the base of data for the rate constants of $S_{1}$ level deactivation and depletion ${ }^{4,13}$ show that quantum yield of dimerization in the case of irradiation of the separate isolated bases should decrease from the intensity of $I=5 \cdot 10^{13} \mathrm{~W} / \mathrm{m}^{2}$ (dotted curve in Figure 2). 


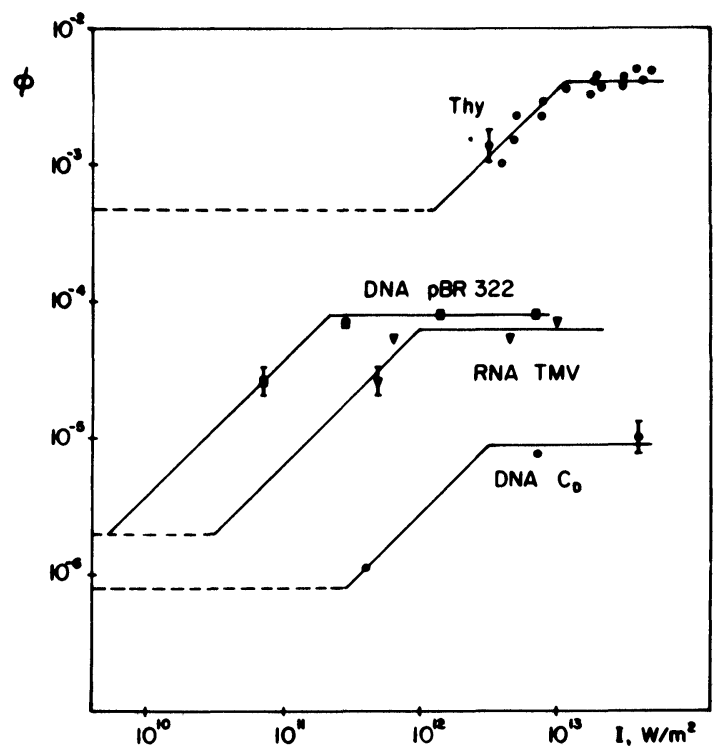

FIGURE 1 Experimental dependences of quantum yields of different photoreactions on the intensity of picosecond laser UV irradiation $\left(\lambda=266 \mathrm{~nm}, I=10^{1{ }^{1}}-10^{14} \mathrm{~W} / \mathrm{m}^{2}\right)$ : single-strand breaks formation in plasmid pBR 322 DNA (squares), induction of breaks of sugar-phosphate chain in TMV RNA (triangles), intramolecular cross-links formation in phage $C_{D}$ DNA (black cricles), photolysis of thymine (open circles). Dotted curves represent the corresponding values of quantum yields under low-intensity continuous UV irradiation $\left(\lambda=254 \mathrm{~nm}, I=10^{\circ} \mathrm{W} / \mathrm{m}^{2}\right)$.

A qualitative interpretation of the effect discovered appears to be the following. To realize two-step excitation the isolated base molecule should absorb two radiation quanta, which can be fulfilled at definite radiation intensities. At the same time the base molecule in a biopolymer may obtain two quanta at considerably small radiation intensities via effective singlet-singlet migration of electronic excitation along a biopolymer chain and following annihilation. This cause to the fact, that in case of biopolymer irradiation the quantum yields of two-quantum photoreactions saturate and the quantum yields of onequantum photoreactions fall at sufficiently lower intensities, than in case of irradiation of the isolated base molecules. 


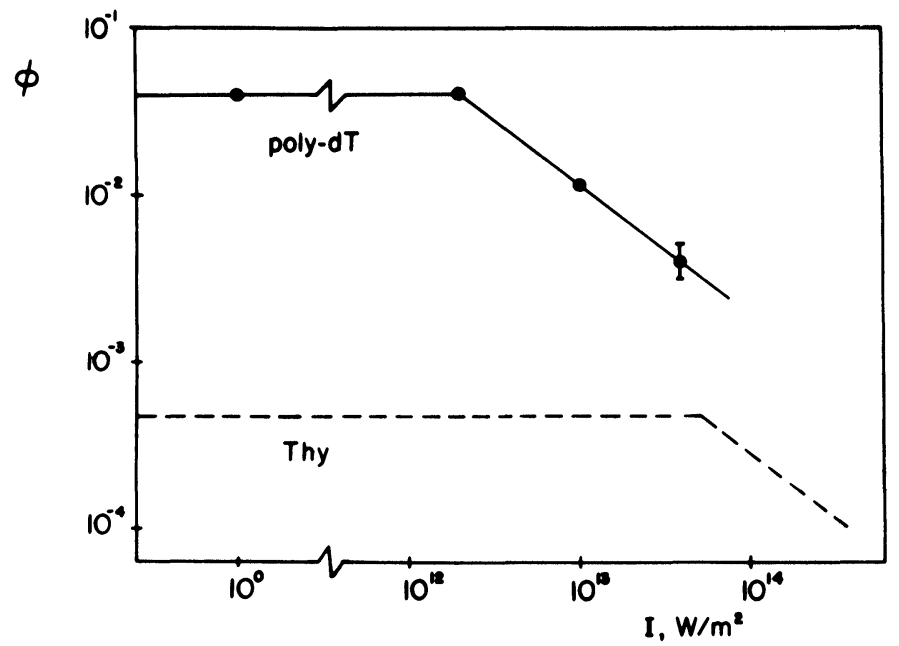

FIGURE 2 Experimental dependence of quantum yield of thymine dimers formation in poly- $d T$ on radiation intensity (solid curve). Dotted curve represents the calculated dependence of the dimerization quantum yield in the case of irradiation of isolated thymine molecules.

\section{Acknowledgements}

The authors express their appreciation to Professor V. S. Letokhov for support of this paper.

\section{REFERENCES}

1. G. J. Fisher and H. E. Johns, in: Photochemistry and Photobiology of Nucleic Acids, Vol. 1, ed. S. Y. Wang (Academic Press, New York, San Francisco, London, 1976), pp. 169, 226.

2. P. G. Kryukov, V. S. Letokhov, D. N. Nikogosyan, A. V. Borodavkin, E. I. Budowsky and N. A. Simukova, Chem. Phys. Lett. 61, 375 (1979).

3. N. A. Simukova, T. N. Menshonkova, E. I. Budowsky and L. B. Rubin, Dokl. Acad. Nauk SSSR, 250, 1398 (1980).

4. D. N. Nikogosyan, D. A. Angelov and A. A. Oraevsky, Photochem. Photobiol. 35, 627 (1982).

5. L. B. Rubin, T. N. Menshonkova, N. A. Simukova and E. I. Budowsky. Photochem. Photobiol. 34, 339 (1981).

6. K. C. Smith and P. C. Hanawalt, Molecular Photobiology (Academic Press, New York and London, 1969).

7. D. N. Nikogosyan, G. G. Gurzadyan and G. B. Zavilgelsky, Dokl. Acad. Nauk SSSR, 269, 485 (1983). 
8. D. A. Angelov, P. G. Kryukov, V. S. Letokhov, D. N. Nikogosyan and A. A. Oraevsky, Kvant. Electr. (Moscow), 7, 1304 (1980).

9. G. G. Gurzadyan, D. N. Nikogosyan, P. G. Kryukov, V. S. Letokhov, T. S. Balmukhanov, A. A. Belogurov and G. B. Zavilgelsky, Photochem. Photobiol. 33, 835 (1981).

10. G. G. Gurzadyan, D. N. Nikogosyan, T. S. Balmukhanov and G. B. Zavilgelsky, Photobiochem. Photobiophys. 4, 87 (1982).

11. H. Boedtker, J. Mol. Biol. 35, 61 (1968).

12. G. B. Zavilgelsky, O. F. Borisova, L. E. Minchenkova and E. E. Minyat, Biokhimiya (Moscow), 29, 508 (1964).

13. M. Daniels, in: Photochemistry and Photobiology of Nucleic Acids, Vol. 1, Ed. S. Y. Wang (Academic Press, New York, San Francisco, London, 1976), p. 84. 